\title{
Synthetic magnetic fields for ultracold neutral atoms
}

Authors: Y.-J. Lin, R. L. Compton, K. Jimenez-Garcia, J. V. Porto, \& I. B. Spielman,

Nature, vol. 462, 628 December(2009).

\section{Recommended with a Commentary by Tin-Lun Ho, Ohio State University}

Recently, the experimental group of Ian Spielman at NIST has produced synthetic gauge fields for cold atoms that act like electro-magnetic fields for charged particles. By applying these gauge fields to a Bose-Einstein condensate, the NIST group was able to generate up to ten vortices, very much like generating vortices in a superconductor using magnetic fields. This is an exciting development in cold atom research since it allows one to simulate transport phenomena in electron systems with cold atoms.

What is surprising is that the experimental set up needed to generate the gauge field is exceedingly simple, shown schematically in Figure 1. It consists of two lasers directed at a condensate of spin-1 Bose atoms $\left({ }^{87} R b\right)$ from opposite directions along $\hat{\mathbf{x}}$, in the presence of magnetic field along $\hat{\mathbf{y}},\left(B_{o}+G y\right) \hat{\mathbf{y}}$, which has a linear field gradient. The momentum and frequency differences of the two lasers ( $q \hat{\mathbf{x}}$ and $\omega$ ) are adjusted so as to induce Raman transitions between successive spin states ( $m=1,0,-1)$, which requires $\hbar \omega=\gamma B_{o}$, where $\gamma$ is the magnetic moment. With $q$ and $\omega$ chosen to meet this specification, vortices are nucleated as the lasers are switched on, (See Figure B). This is truly remarkable in view of the challenges encountered in the conventional method of generating vortices, which requires the confining traps with almost perfect cylindrical symmetry, and with angular momentum in perfect alignment its symmetry axis. In contrast, the confining trap here can be of arbitrary shape, and no physical rotation is required.

The origin of this gauge field is Berry phase. The idea is that in the presence of a spatially varying magnetic field $B_{\text {eff }}(\mathbf{r})$, the kinetic energy of spin state $|1,1\rangle=\left|S=1, \hat{\mathbf{B}}_{\text {eff }}(\mathbf{r}) \cdot \mathbf{S}=1\right\rangle$ aligned with the local field is of the electro-magnetic form $(\mathbf{p}-\mathbf{A})^{2} / 2 M$, where $\mathbf{A}(\mathbf{r})=-i \hbar\langle 1,1|\nabla| 1,1\rangle$ is the Berry phase due to the spatial variation of 1,1$\rangle$. What the set up in Figure $\mathrm{A}$ does is to produce an effective field $\mathbf{B}_{\text {eff }}(\mathbf{r})$ which give rise to a 

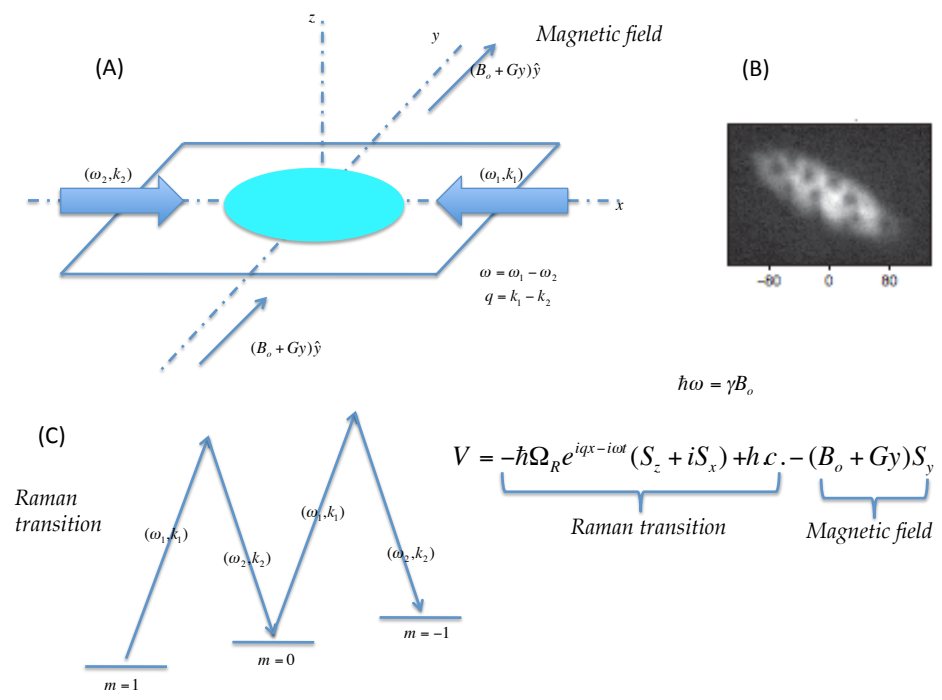

Figure 1: The Experimental set up and other aspects discussed in the text

vector potential with sufficiently large rotation $\nabla \times \mathbf{A}$. It works as follows. The Raman transition and the non-uniform field gives rise to an interaction $V=-\hbar \Omega_{R} e^{i q x-i \omega t}\left(S_{z}+i S_{x}\right)+h . c .-\gamma\left(B_{o}+G y\right) S_{y}$, where $\Omega_{R}$ is the strength of the Raman process, which changes the spin along $\hat{\mathbf{y}}$ while transferring momentum $q \hat{\mathbf{x}}$ to the system. (See Figure C). At resonance, $\hbar \omega=\gamma B_{o}$, $V$ becomes a static interaction $\bar{V}=-\hbar \Omega_{R} e^{i q x}\left(S_{z}+i S_{x}\right)+$ h.c. $-G y S_{y}$ in the frame rotating along $\hat{\mathbf{y}}$ with frequency $\omega$, and is exactly of the form $\bar{V}=-\mathbf{B}_{e f f}(\mathbf{r}) \cdot \mathbf{S}$, where $\mathbf{B}_{e f f}(\mathbf{r})$ is rotating in the $x z$ plane as one moves along the direction, and points along $\hat{\mathbf{y}}$ and $-\hat{\mathbf{y}}$ and $y$ as approaches $+\infty$ and $-\infty$. As it turns out, this non-uniform magnetic field is able to generate a vector potential with sufficiently large rotation to produce vortices.

The fact that vortices can be generated with such great ease raises the hope that this may be the path to realizing quantum Hall states. At present, experimental groups at NIST and ENS (Paris) are trying to create gauge fields of great strength. Should the quantum Hall state be realized in cold atoms, it will surely generate a great deal of excitement, for not only shall we add to the electron quantum Hall state a bosonic counterpart, but also 
open new doors for realizing quantum Hall states of high spin particles, since many atoms have large spins. At the moment, there is also considerable experimental interest in generating non-abelian gauge fields. It is conceivable that we may see experimental reports about this in the near future. 